This item was submitted to Loughborough's Research Repository by the author.

Items in Figshare are protected by copyright, with all rights reserved, unless otherwise indicated.

\title{
The ranking of negative-cost emissions reduction measures
}

PLEASE CITE THE PUBLISHED VERSION

http://dx.doi.org/10.1016/j.enpol.2012.05.071

PUBLISHER

(c) Elsevier

VERSION

AM (Accepted Manuscript)

LICENCE

CC BY-NC-ND 4.0

REPOSITORY RECORD

Taylor, Simon. 2019. "The Ranking of Negative-cost Emissions Reduction Measures". figshare. https://hdl.handle.net/2134/13076. 
This item was submitted to Loughborough's Institutional Repository (https://dspace.lboro.ac.uk/) by the author and is made available under the following Creative Commons Licence conditions.

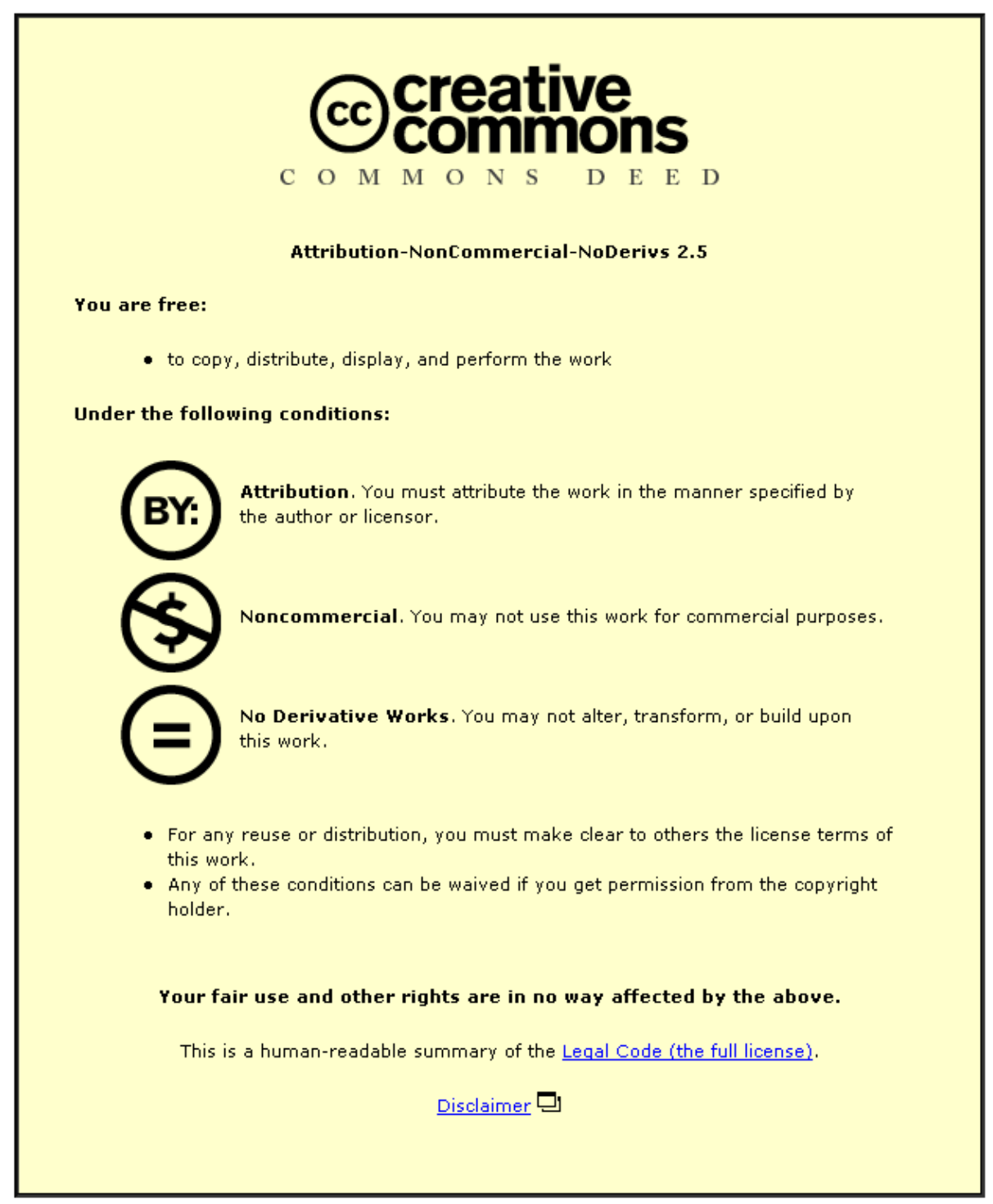

For the full text of this licence, please go to: http://creativecommons.org/licenses/by-nc-nd/2.5/ 
NOTICE: this is the author's version of a work that was accepted for publication in Energy Policy. Changes resulting from the publishing process, such as peer review, editing, corrections, structural formatting, and other quality control mechanisms may not be reflected in this document. Changes may have been made to this work since it was submitted for publication. A definitive version was subsequently published in Energy Policy, vol. 48, pp. 430-438 (2012),

http://dx.doi.org/10.1016/j.enpol.2012.05.071

\title{
The ranking of negative-cost emissions reduction measures
}

\author{
Simon Taylor \\ School of Civil and Building Engineering, \\ Loughborough University, UK \\ LE11 3TU \\ s.c.taylor@lboro.ac.uk $+44(0) 1509228788$
}

\begin{abstract}
A flaw has been identified in the calculation of the cost-effectiveness in marginal abatement cost curves (MACCs). The problem affects "negative-cost" emissions reduction measures - those that produce a return on investment. The resulting ranking sometimes favours measures that produce low emissions savings and is therefore unreliable. The issue is important because incorrect ranking means a potential failure to achieve the best-value outcome. A simple mathematical analysis shows that not only is the standard cost-effectiveness calculation inadequate for ranking negative-cost measures, but there is no possible replacement that satisfies reasonable requirements. Furthermore, the concept of negative cost-effectiveness is found to be unsound and its use should be avoided. Among other things, this means that MACCs are unsuitable for ranking negative-cost measures. As a result, MACCs produced by a range of organizations including UK government departments may need to be revised. An alternative partial ranking method has been devised by making use of Pareto optimization. The outcome can be presented as a stacked bar chart that indicates both the preferred ordering and the total emissions saving available for each measure without specifying a cost-effectiveness.
\end{abstract}

\section{Keywords}

Emissions

Abatement

Cost-effectiveness 


\section{Introduction}

Marginal abatement cost curves (MACCs) are a common method of assessing the economics of measures to reduce emissions of greenhouse gases, particularly carbon dioxide. They first appeared about twenty years ago (Jackson and Roberts 1989, Jackson 1991, Mills et al 1991, Sitnicki et al 1991) as a variation on the energy conservation supply curves introduced by Meier (1982). In spite of some doubts about the behaviour of the calculations for negative costs (Wallis 1992a, 1992b; Jackson 1992, 1993), the use of such curves has become widespread in a variety of contexts. They have been applied to specific sectors such as non-domestic buildings (Pout 2000), waste (Beaumont and Tinch 2004, Hogg et al 2008), transport (Spencer and Pittini 2008), higher education (SQW Energy 2009) and the National Health Service in England (Hazeldine et al 2010). They have also been applied to whole countries, e.g. Denmark (Morthorst 1994), the UK (Enviros 2006, Toke and Taylor 2007, DTI 2007, CBI 2007, CCC 2008), and the USA (Creyts et al 2007, Bloomberg 2010). In addition, the McKinsey company (McKinsey 2012) has produced MACCs for a large number of countries including Australia, Belgium, Brazil, China, Czech Republic, Germany, India, Israel, Poland, Russia, Sweden and Switzerland. MACCs have also been applied to non- $\mathrm{CO}_{2}$ emissions (EPA 2006), and their use recently extended to forming the basis for carbon pricing by the British government (DECC 2009). Recent examples include their use in the British Government's Green Deal programme (DECC 2011). Using the useful nomenclature of Kesicki (2010), the present work focuses on such "expert-based" curves which deal with individual measures, rather than "model-derived" ones. Kesicki and Strachan (2011) analysed some disadvantages relating to MACCs and pointed out that little academic work has 
been carried out on them. Kesicki and Ekins (2012) went further by calling for caution by policymakers in the use of MACCs.

All methods of constructing these curves work in roughly the same way. Each emissions reduction measure is assigned two values: a figure of merit which indicates its cost-effectiveness in $£ / t \mathrm{C}$ (tonne of carbon) or equivalent, and the total emissions reduction achievable over the period of interest. A rectangular block is plotted for each measure with height and width respectively corresponding to these values. The blocks are lined up from the smallest on the left to the largest on the right and the optimum outcome is obtained by implementing the measures in order from left to right. The total width of the blocks indicates the total emissions reductions achievable.

Some measures have negative costs, which means that their implementation results in a net profit over the period of interest. A typical example is domestic insulation which typically pays for itself within a few years. In such cases, the blocks extend below the $x$-axis and the most negative measure is taken to represent best value. So according to the MACC of Toke and Taylor (2007) in Fig. 1, the domestic lighting measure should be implemented first, followed by reduction of standby drain and so on.

On the face of it, it seems surprising that such negative-cost measures exist. One might expect that the availability of, effectively, something for nothing would cause the measures to be quickly taken up and the potential exhausted, leaving MACCs to deal only with positive costs. As Kesicki and Ekins (2012) put it, “This phenomenon ... is not compatible with an efficient market." Explanations for this inertia include consumers' aversion to perceived debt and ability to cope with a limited amount of 
information at one time (DECC 2011). In addition, Kesicki and Ekins' analysis of negative abatement costs concluded that the negative abatement potential is often overstated. The present work is not concerned with such arguments but simply with the treatment of negative abatement potential as it appears in the vast majority of published MACCs.

The cost-effectiveness of emissions reduction measures is also determined outside the framework of MACCs. For example, values of the cost-effectiveness of behavioural interventions were reported by Allcott and Mullainathan (2010) and calculations for transport measures were carried out by Kok et al (2011).

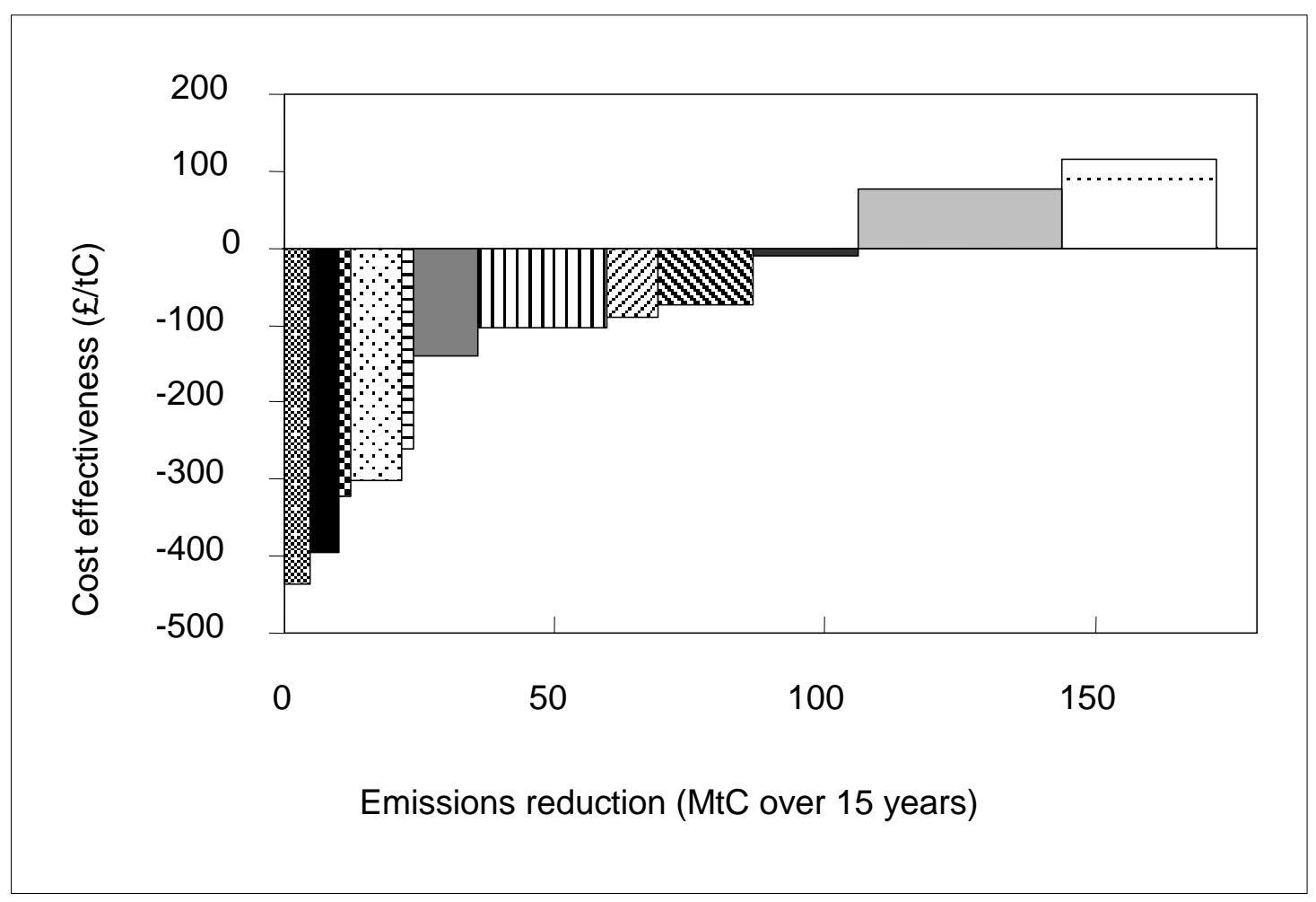

\begin{tabular}{|c|c|c|c|}
\hline 原 & Domestic lighting & Non-domestic lighting & Renewables \\
\hline & Reduction of standby drain & Motors & Nuclear / Revised Oxera \\
\hline $\mathbf{8}$ & Savaplugs & Domestic insulation & Nuclear / NEF \\
\hline$\because \because$ & No new electric heating & BEMS & \\
\hline 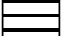 & Compressed air leaks & $\mathrm{CHP}$ & \\
\hline
\end{tabular}

Figure 1. MACC of Toke and Taylor (2007) 
The issue addressed in this paper is the validity of the standard figure of merit for cost-effectiveness, measured in $£ / t C$ or similar units and here denoted $M_{s t d}$, when the costs are negative.

The structure of the paper is as follows. Section 2 describes a serious problem with the standard way of calculating cost-effectiveness (which will be referred to as the standard metric or standard figure of merit) when applied to emissions-saving measures that make a return on investment. An analysis in Section 3, supported by a simple mathematical proof in Appendix A, shows that no metric satisfying reasonable requirements is possible for such measures. An alternative ranking method based on Pareto optimization is proposed in Section 4 and applied to some existing results in Section 5, revealing ranking errors by the standard metric. A discussion in Section 6 covers such issues as consequences for existing results and options for dealing with MACCs in the future. It also examines why the problem has not been addressed before now. Finally, conclusions are drawn in Section 7 .

\section{The problem}

The standard figure of merit can be calculated in a variety of ways. The method used in the author's previous work (Toke and Taylor 2007), following Jackson and Roberts (1989), was to determine the cost of the measure in $£$ per MWh saved and divide by the mass of emissions per MWh saved. The cost was calculated as the net present value (NPV) of the measure using the same discount rate and period (5\% and 15 years in this case) for all measures considered, and divided by the energy saved over the period of interest to give the required numerator. Equivalent methods, e.g. Bloomberg (2010), measure costs in \$/year and emissions savings in tC/year. It is convenient to assume for the present work that the normalizing value is energy 
saved, and to refer to the relevant quantities as specific costs (Blok et al 1993) and specific emissions savings. So the standard metric can be described as

$$
M_{s t d}=\frac{c}{g}
$$

where $c=$ specific cost and $g=$ specific emissions saving.

The specific emissions saving $g$ is always positive for the measures of interest, so when the specific cost $c$ is positive, corresponding to a net financial loss, so is $M_{s t d}$. A smaller $M_{s t d}$ is obtained from a lower specific cost or a larger specific emissions saving or both. Lower cost and higher emissions savings are both desirable objectives, so for positive costs the measure with the smallest $M_{\text {std }}$ provides the smallest outlay per unit mass of emissions saved, and therefore the best value.

However, when the specific cost is negative (corresponding to a net return on investment, or profit), the picture changes. A smaller (i.e. more negative) $M_{s t d}$ is achieved by a greater financial return, which is a desirable objective, or by a reduction in the specific emissions saving, the opposite of what is desired. This means that the measure with the lowest $M_{\text {std }}$ is not necessarily the best option. More generally, it means that the ranking of a set of negative-cost measures, like that in Fig. 1, is not reliable. The problem is a serious one because an incorrect ranking means a potential failure to achieve the best-value outcome.

A simple example demonstrates the problem. Suppose there is a plan to install insulation in one or other of two identical houses, one heated by gas and the other by electricity. Insulation is simply a method of reducing heat loss, so the details, and in particular the capital and installation costs, are independent of the fuel. Now, for the 
purposes of illustration, suppose the cost of electricity is the same as that of gas. Then at current prices the NPV of the measure over a reasonable time period - say 15 years - will be positive (that is, there will be a net return) and the same for both houses. The amounts of heat saved over this period will also be equal, so the cost per unit energy saved, $c$, will be the same negative value in both cases.

The figure of merit $M_{s t d}$ is $c$ divided by the mass of emissions per unit energy saved, which in this case is simply the emissions factor for the fuel. That for gas is currently less than half that for UK grid electricity, making the figure of merit for the gasheated house more negative. So according to the standard approach, insulation of the gas-heated house is preferred, although the financial position is identical and the emissions saving considerably worse than for the electrically-heated house.

To press the point home, if a third house used a very low-carbon heating technology at the same fuel cost, it would yield a smaller value still of $M_{s t d}$ and would therefore be preferred over the gas-heated case, even though the emissions savings would be negligible.

\section{Analysis}

Although the conventional figure of merit $M_{\text {std }}$ defined in equation (1) works correctly when the specific cost is positive, it is clear that there is a problem when it is negative.

The switch in the way $M_{s t d}$ responds to the emissions saving when the cost changes sign is a consequence of its functional form as shown in Fig. 2. First, note that in 
one respect the function always behaves correctly: a reduction in specific $\operatorname{cost} c$ at constant $g$ always leads to a decrease in $M_{\text {std }}$, as shown by arrow 1 .

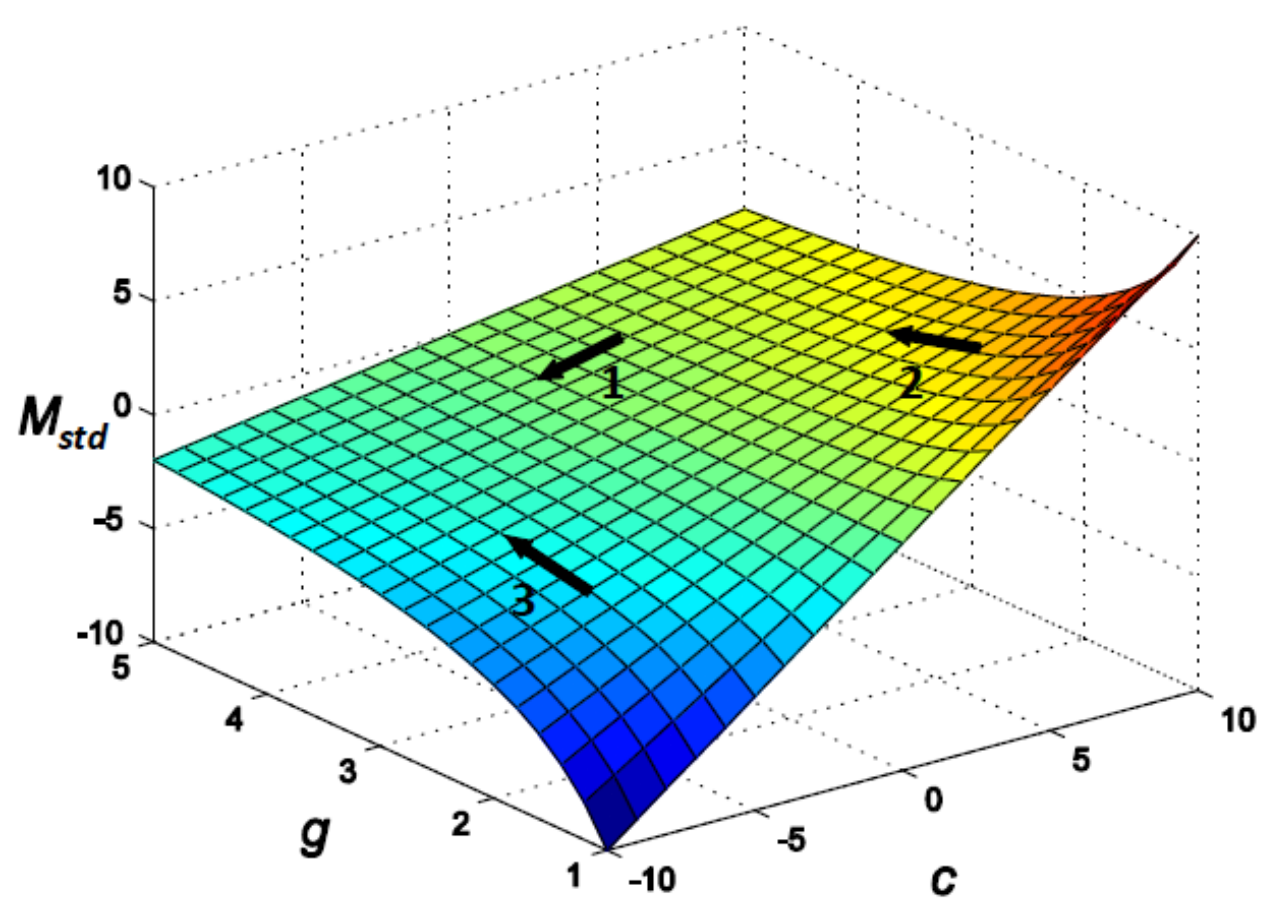

Figure 2. Surface plot of metric $M_{\text {std }}$ as a function of specific cost $c$ and specific emissions saving $g$

When $c>0$, corresponding to a positive specific cost, the function also behaves in the required way: an increase in the specific emissions saving $g$ always leads to a decrease in $M_{\text {std }}$ as shown by the downhill-pointing arrow 2 . If the function behaved correctly, an increase in $g$ would also lead to a decrease in $M_{s t d}$ when $c<0$, so that arrow 3 pointed downhill in the same way as arrow 2. But as shown in the figure, arrow 3 points uphill, which means that an increase in the specific emissions saving $g$ causes an increase, not a decrease, in $M_{s t d}$.

The problem cannot be solved by minor adjustments in the calculation, such as ignoring minus signs. The function is fundamentally inappropriate when the costs 
are negative. What is needed is a metric that when applied to the negative $c$ domain behaves like arrows 1 and 2. This need for the correct slopes in the figure of merit will be referred to as Requirement 1 .

From a purely mathematical point of view (and so ignoring what the expressions might mean), it is possible to conceive of alternative functions of $c$ and $g$ that satisfy Requirement 1 in the negative $c$ domain. One in particular will be considered now to bring out another important issue. Let the new metric be defined as

$$
M_{a l t}=c g \text {, }
$$

that is, the product of specific cost and specific emissions saving rather than their quotient. Equation (2) is consistent with Requirement 1 when $c$ is negative in that greater financial returns and greater emissions savings are both favoured. (Its behaviour elsewhere is not consistent but that is not relevant for the present argument.)

However, this metric has another feature that deserves attention. Suppose it was applied to two different measures, one (A, say) with a very negative value of $c$ and a low value of $g$, and the other (B) with a slightly negative value of $c$ and a high value of $g$, such that the product $M_{\text {alt }}$ was the same in both cases. The logic of the process is that these two measures should have equal ranking. But it is clear that measure A would be favoured by an investor whose sole desire was a high financial return while B would be favoured by an environmentalist whose priority was to reduce carbon emissions. 
The point becomes even sharper in the case of one of the measures having a marginally advantageous value of $M_{\text {alt }}$ because the other would still be preferred by its supporter, thus making the figure of merit redundant.

The potential for such disagreement demonstrates that the metric $M_{a l t}$ is unsuitable for the purpose of ranking. This need for incontestability will be referred to hereafter as Requirement 2.

$M_{a l t}$ is contestable because the same value can be obtained using different sets of values of $c$ and $g$, such that one set would be preferred by an investor and another by an environmentalist. A necessary (though possibly not sufficient) condition to satisfy Requirement 2 is that $M$ corresponds to a fixed ratio of $c$ to $g$. Since the ratio for a given value of $M$ cannot vary, there can be no disagreement. This means that the functional form must be $M=f(c / g)$.

Although other requirements for a suitable metric could be proposed, the two considered so far allow a fundamental conclusion to be drawn. A mathematical statement of the proof is provided in Appendix A, but the argument can be simply stated. Either the metric has a functional form $M=f(c / g)$ or it has not. If it has, it fails to satisfy Requirement 1 (correct slopes) in the negative $c$ domain; if it has not, it fails to satisfy Requirement 2 (contestability). So no metric can simultaneously satisfy Requirements 1 and 2 in the negative $c$ domain. In less formal language, no figure of merit satisfying reasonable requirements is possible for negative-cost measures.

\section{An alternative ranking method}


Although the proof in the previous section rules out figures of merit for ranking emissions reduction measures, alternative approaches exist. One such is a method of multi-objective optimization making use of the Pareto front (e.g. Deb 2001). In the present case the Pareto front is determined repeatedly in such a way as to produce a ranking order for sets of emissions reduction measures. This method of "nondominated ranking", introduced by Goldberg (1989), will be referred to as "Pareto ranking" for simplicity, although other Pareto-based approaches are possible, e.g. Fonseca and Fleming (1995), Deb (2001).

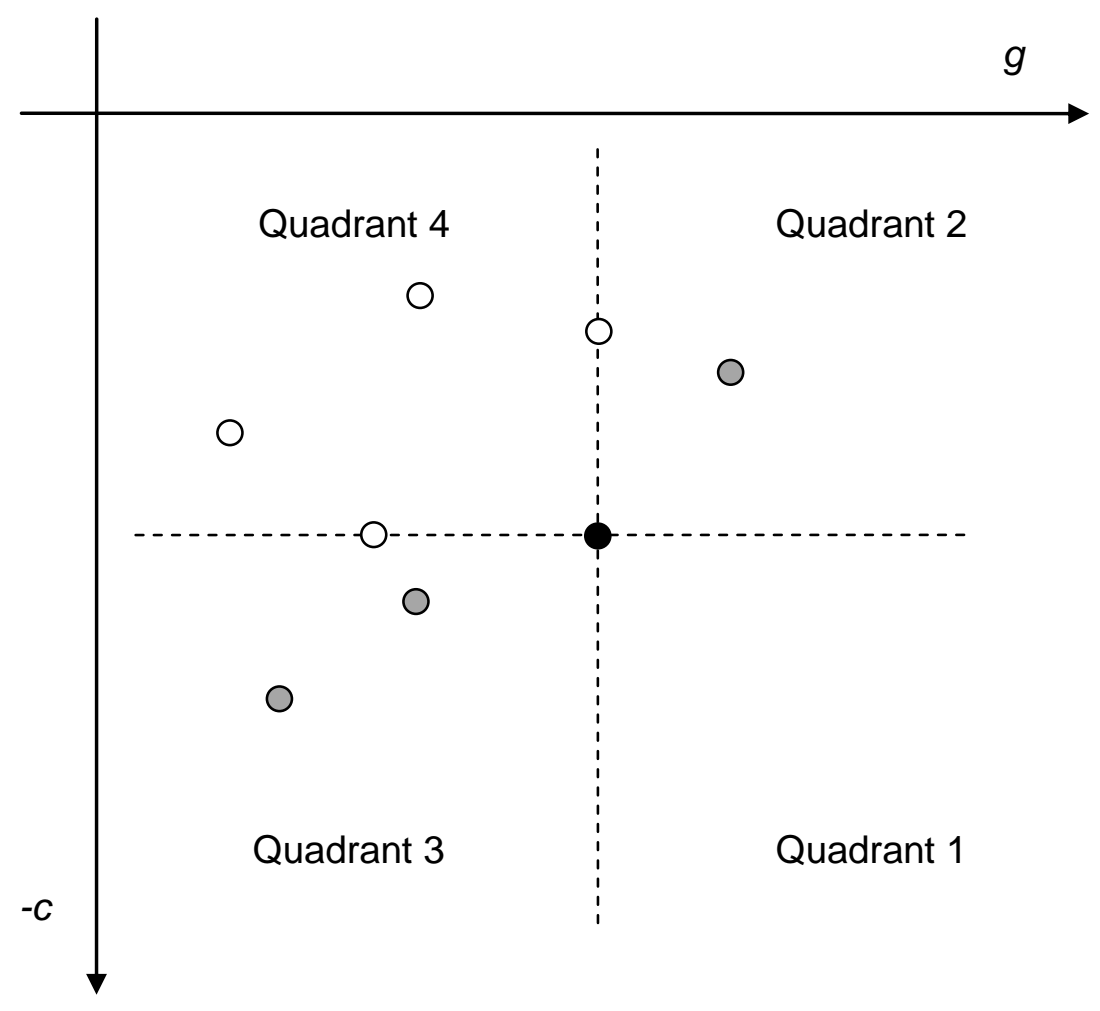

Figure 3. Definition of a Pareto front for emissions reduction measures

The method is illustrated by plotting the $c$ and $g$ values of a fictitious set of negativecost measures in Figure 3. The proposed approach considers the relative positions of the points, each of which represents a different measure. A better emissions 
performance corresponds to a larger (more positive) value of $g$, and a better financial outcome to a smaller (more negative) value of $c$.

The definition of a Pareto front is simplified by introducing the concept of dominance. In the context of the present work, measure A dominates measure $\mathrm{B}$ if $c_{A}$ $<c_{B}$ and $g_{A} \geq g_{B}$, or $c_{A} \leq c_{B}$ and $g_{A}>g_{B}$; that is, if the financial outcome or the emissions performance of $\mathrm{A}$ is better than that of $\mathrm{B}$ and the other is no worse. In Figure 3, the measure represented by the black point dominates all the measures represented by white points in Quadrant 4, including those on the borders defined by the dotted lines. It neither dominates nor is dominated by the grey points in Quadrants 2 and 3. If there were any points in Quadrant 1, including on the dotted boundaries, then the black point would itself be dominated.

If such a comparison is applied to all of the measures of interest (namely those with a negative cost), each measure will end up being either (a) dominated by one or more measures or (b) not dominated. In terms of Fig. 3, this test corresponds to placing the framework of dotted lines on each point and checking whether any of the other points fall in its Quadrant 1. Measures that are not dominated are referred to as Pareto-optimal and form a Pareto front, behind which the dominated measures lie. For the set of measures in Figure 3, the Pareto front consists of the black and grey points.

The Pareto front consists of those measures that have a combination of cost and emissions performance that puts them ahead of the rest, allowing them to be justifiably ranked first. There are two important points to note. First, this approach offers no way of distinguishing between the measures that comprise the Pareto front: 
they must be ranked together. Second, it does not follow that every measure in the Pareto front dominates all the lower-ranked measures.

The next stage of the ranking process is to remove the points comprising the front and then to repeat the procedure with the points that remain. This will identify a new Pareto front that will include all measures that are second best.

If this process is continued until all points have been accounted for, a ranking will be defined that, while less discriminating than a metric (because sets of measures of equal ranking are defined), provides a clear order.

\section{Numerical comparisons}

\subsection{Application to data of Toke and Taylor (2007)}

In order to demonstrate the use of the Pareto ranking it has been applied to the negative-cost subset of the measures considered by Toke and Taylor (2007), for which the underlying data are known. This is done for the purposes of illustration only, since the passage of time since that study means that the results now have limited relevance.

Table 1 lists a set of emissions-reducing measures that provide a return on investment. The values of $c$ and $g$ and the resulting standard figure of merit $M_{s t d}$ are given for each along with the ranking it produces. Alongside is listed the ranking obtained by applying the Pareto method. Rankings containing " =" contain sets of equally-ranked measures that appear in the same Pareto front. The items ranked 1 represent the optimal emissions reduction measures according to the corresponding method. 
Table 1. Rankings for a set of measures taken from Toke and Taylor (2007)

\begin{tabular}{|c|c|c|c|c|c|}
\hline & \multirow{2}{*}{$\begin{array}{l}\text { Specific } \\
\text { cost } \\
\text { saving } c \\
£ / \mathrm{MWh}\end{array}$} & \multirow{2}{*}{$\begin{array}{c}\text { Specific } \\
\text { emissions } \\
\text { saving } g \\
\text { kgC/MWh }\end{array}$} & \multirow{2}{*}{$\begin{array}{l}M_{s t d} \\
£ / t C\end{array}$} & \multicolumn{2}{|c|}{ Ranking } \\
\hline & & & & $M_{\text {std }}$ & Pareto \\
\hline A. Domestic lighting & -54.02 & 124.1 & -435 & 1 & 1 \\
\hline B. Reduction of standby drain & -49.11 & 124.1 & -396 & 2 & 2 \\
\hline C. Savaplugs & -39.99 & 124.1 & -322 & 3 & 3 \\
\hline D. No new electric heating & -21.83 & 72.3 & -302 & 4 & $6=$ \\
\hline $\mathrm{E}^{\mathrm{a}}$. Insulation in gas-heated homes - eq. fuel cost & -15.41 & 51.8 & -297 & 5 & $9=$ \\
\hline F. Compressed air leaks & -32.43 & 124.1 & -261 & 6 & 4 \\
\hline$G^{a}$. Insulation in electrically-heated homes - std. fuel cost & -29.95 & 124.1 & -241 & 7 & 5 \\
\hline H. Non-domestic lighting & -17.21 & 124.1 & -139 & 8 & $6=$ \\
\hline$I^{a}$. Insulation in electrically-heated homes - eq. fuel cost & -15.41 & 124.1 & -124 & 9 & 8 \\
\hline J. Motors & -12.92 & 124.1 & -104 & 10 & $9=$ \\
\hline K. Domestic insulation & -5.65 & 62.7 & -90 & 11 & 11 \\
\hline L. BEMS & -3.83 & 51.8 & -74 & 12 & 12 \\
\hline $\mathrm{M}^{\mathrm{a}}$. Insulation in gas-heated homes - std. fuel cost & -1.37 & 51.8 & -26 & 13 & 13 \\
\hline N. CHP & -0.25 & 25.0 & -10 & 14 & 14 \\
\hline
\end{tabular}

a. Additional insulation examples

Domestic insulation was dealt with in Toke and Taylor (2007) as measure K, which represents the economics and emissions savings for average UK homes with $15 \%$ of the heating by electricity and the rest by gas. Such an approach masks the issues being studied in the present paper, so some variations have been included, indicated by a superscript "a" in Table 1 . First, the domestic insulation calculations have been applied separately to electric and gas-heated homes in measures $\mathrm{G}$ and $\mathrm{M}$ using the standard fuel prices at the time. Second, the calculations have also been performed for the two types of heating on the basis of an equal, roughly average, fuel cost in measures $\mathrm{E}$ and $\mathrm{I}$, thus mirroring the description in the introduction. Making the specific costs of insulation equal in this way clarifies the impact of variations in the specific emissions saving. 
The first point to note relates to the ranking by the standard metric $M_{s t d}$. Of the two additional insulation examples with equal fuel costs, measure $\mathrm{E}$ for gas-heated homes which makes lower emissions savings is ranked well above measure I for electricity. This is in line with the earlier discussion and is strong evidence for the inapplicability of the metric due to its failure to satisfy Requirement 1 .

The Pareto ranking shifts the gas insulation measure E below the electrical one I, in line with its lower emissions savings and therefore poorer cost-effectiveness. It also demotes the "No new electric heating" measure D from fourth to equal sixth place. The key difference between D and E and those above them in the Pareto ranking is the specific emissions saving. It is around half that of the other measures, in the first case because gas emissions are being saved and in the second because electricity emissions are being replaced by gas. Their anomalously high ranking by the standard metric $M_{s t d}$ is a consequence of its incorrect behaviour.

If the additional examples are excluded, only the positions of measures $\mathrm{D}, \mathrm{F}$ and $\mathrm{H}$ change as a result of the Pareto ranking. Measure F (Compressed air leaks) moves up and $\mathrm{D}$ (No new electric heating) moves down to become level with $\mathrm{H}$ (Non-domestic lighting). Thus even with an apparently flawed figure of merit, results consistent with the Pareto ranking were produced by $M_{s t d}$ in most cases. In particular, for the additional insulation examples with standard fuel costs, $M_{\text {std }}$ sets the electricallyheated case $\mathrm{G}$ above the gas-heated one M. The reason for this correct ordering is that the price of electricity exceeds that of gas by a factor of about four, and the cost savings outweigh the perverse effect of the lower emissions factor. 


\subsection{Approximate calculations suggesting incorrect rankings}

In order to determine a Pareto ranking for a set of emissions reduction measures the values of $c$ and $g$ for each need to be known. This presents a difficulty for published data because these values are not usually available. In both of the cases described below, however, it was possible to deduce values of the emissions factors $g$ for two neighbouring measures in published MACCs. Values of $M_{s t d}$ were read from the published plots and $c$ was then obtained via equation (1). It was then possible to deduce the Pareto ranking of the neighbouring measures and compare it with the positions in the published MACC.

\subsubsection{MACC produced by McKinsey for CBI (2007)}

The MACC produced by McKinsey for the UK for the year 2020 on behalf of the CBI (2007, Fig. 13, p. 44) shows building structure to be sixth in the ranking of measures, with an abatement cost of about $-€ 140 / \mathrm{tCO}_{2} \mathrm{e}$, with lighting in seventh position with a cost of about $-€ 115 / \mathrm{tCO}_{2} \mathrm{e}$. The diagram is reproduced in Fig. 4.

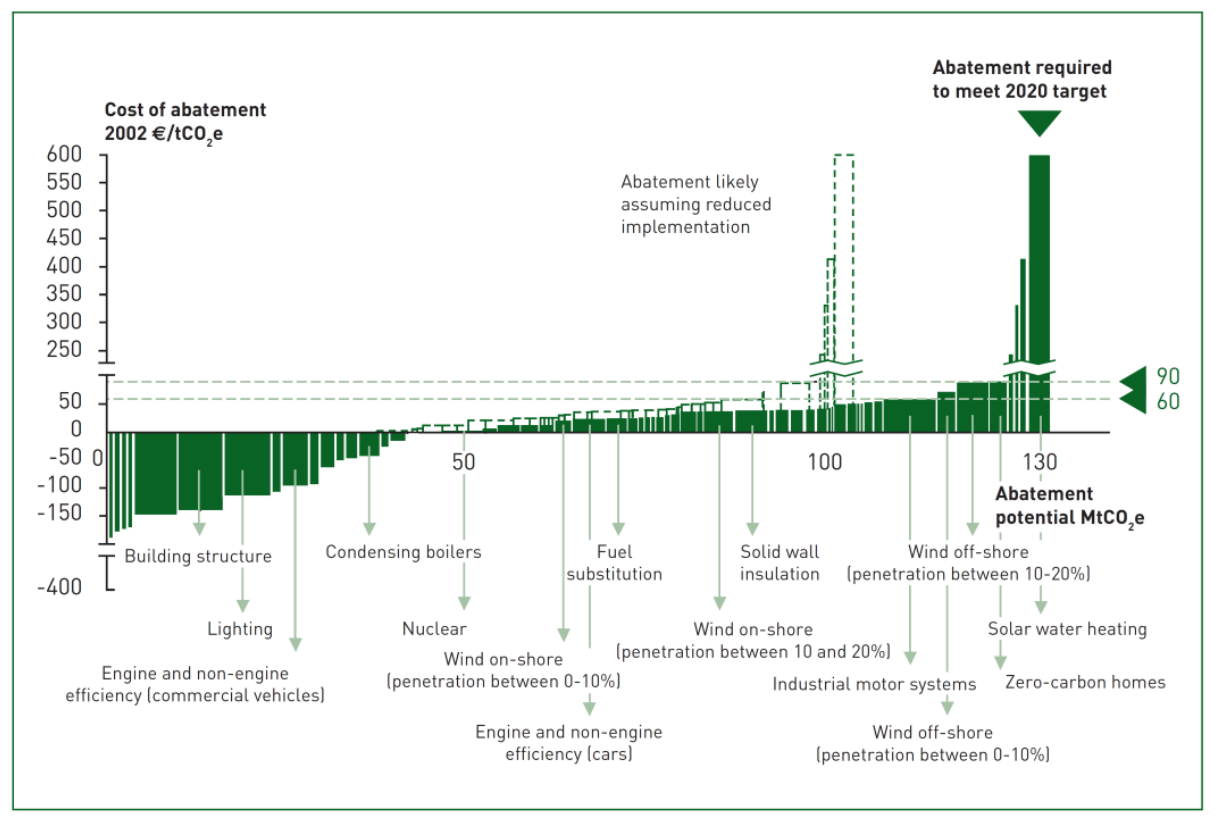

Figure 4. MACC of McKinsey (CBI 2007) 
On the assumption that the effect of building structure changes will be to reduce emissions from heating systems, the specific emissions change will be somewhere between the emissions factor of gas, which in the relevant units is 0.185 $\mathrm{tCO}_{2} \mathrm{e} / \mathrm{MWh}$, and that of electricity at $0.543 \mathrm{tCO}_{2} \mathrm{e} / \mathrm{MWh}($ Defra 2011).

The present argument is best served by an emissions factor close to the lower value, corresponding to a high proportion of space heating by gas, and this would be in line with the current UK value of about $79 \%$ according to DECC (2010). But to ensure the argument does not depend on this figure, it will be supposed that the emissions factor is half-way between those of gas and electricity, giving a specific emissions saving for the building structure measure of $0.364 \mathrm{tCO}_{2} \mathrm{e} / \mathrm{MWh}$.

The corresponding values of $c$ are then $-140 \times 0.364=-€ 50.96 / \mathrm{MWh}$ for building structure and $-115 \times 0.543=-€ 62.45 / \mathrm{MWh}$ for lighting. So the lighting measure makes a bigger financial return and saves more emissions than building structure - in Pareto terms, it dominates - so according to Pareto ranking, the relative positions in the McKinsey ranking are incorrect. The lighting measure is more, not less, cost effective than the building structure measure.

\subsubsection{MACC for non-domestic buildings in Pout (2000)}

A MACC for non-domestic buildings published by Pout (2000) shows that a measure to fit condensing boilers (her item 15) has a net annual cost of carbon saved of about $-£ 200 / \mathrm{tC}$ while replacement of incandescent with compact fluorescent lamps (item 1) has a value of about $-£ 180 /$ tC. So the condensing boiler measure is more cost-effective and is ranked higher. The diagram is reproduced as Fig. 5. There are too many measures to list in full, but they cover lighting changes (1 to 5), localized domestic hot water (6), insulation and glazing (7 to 11), electrical 
equipment efficiency (12, 13 and 16), building energy management systems (14) and condensing boilers (15). Note that some measures appear more than once, due, presumably, to the use of different assumptions.

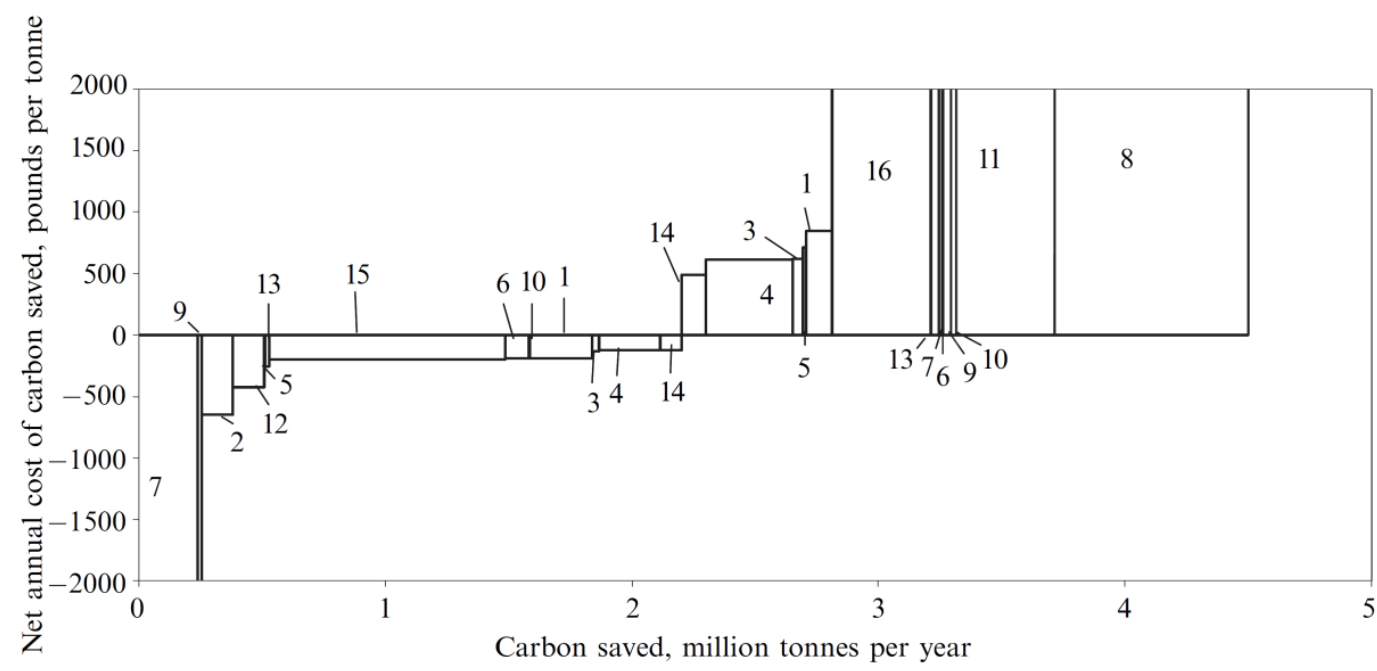

Figure 5. MACC of Pout (2000)

For the lighting measure (item 1), the specific cost can be determined in the same way as in Section 5.2.1 and turns out to be $-180 \times 0.543=-£ 97.74 / \mathrm{MWh}$. For the condensing boiler measure the result depends on what type of heating is being replaced. For the two main possibilities, however, the results are very similar. For replacement of electric heating, the specific cost is $-180 \times(0.364-0.185)=$ $-£ 32.22 / \mathrm{MWh}$ while for replacement of gas it is $-180 \times 0.185=-£ 33.30 / \mathrm{MWh}$. Either way, the lighting measure makes a financial return three times greater and saves twice the emissions and according to Pareto ranking should therefore rank higher, not lower, than the condensing boiler measure. 


\section{Discussion}

\subsection{The existence of a problem}

The analysis of the standard metric $M_{s t d}$ shows that there is no doubt that there is an issue to be addressed. It cannot be right that a method of ranking emissions reduction measures favours lower emissions savings.

One problem is that the metric conventionally used for ranking, known variously as the cost-effectiveness, the "cost of saving $\mathrm{CO}_{2}$ " (Jackson 1989) or the "abatement cost" (Bloomberg 2010), measured in $£ / t C$ or equivalent units, has such a commonsense feel to it that seeking alternatives seems perverse. Of course, where a measure has a positive cost the metric works correctly. Its intuitive meaning fits well with its technical job of ranking measures.

However, when there is a net return on investment, corresponding to a negative value of the metric, the behaviour becomes inappropriate. It is perhaps fortunate that the cost of electricity exceeds that of gas by a factor of about four, while the ratio of the emissions factors is nearer two. This means that the perverse effect is often outweighed and the ranking is accidentally correct. But the examples in Section 4 and 5 show that incorrect rankings can still arise. In addition, future changes in fuel price and the carbon intensity of the grid may make this fortuitous correction less common.

\subsection{The validity of "cost-effectiveness" for profit-making measures}

The favouring by the standard calculation method of measures with small emissions savings raises the question of whether it is meaningful to calculate values of $M_{\text {std }}$ when the specific cost is negative. It is clear that the arithmetic operation is a valid 
one since the metric is simply the quotient of two real numbers. What is more, the quantity calculated is indeed the return on investment per tonne of carbon saved. The key point is that it is not a valid figure of merit since it promotes maximizing the financial return but minimizing the carbon savings. In particular, it is not a measure of cost-effectiveness. For that reason, it is unsuitable for ranking measures that give a return on investment.

The more general point revealed by the analysis is that there is no possible alternative figure of merit based on $c$ and $g$ that satisfies reasonable requirements. Since "merit" in this case must mean "cost-effectiveness" - there is no other criterion of interest if one is discussing the economics of emissions reduction measures - the conclusion must be that negative cost-effectiveness, defined as a numerical value, is not a valid concept.

The proof that no figure of merit exists makes intuitive sense, because no exchange is taking place. It is no longer a case of A giving B some money and in exchange achieving some emissions reductions. Instead, A gets both money and emissions reductions. No single function can satisfy both the investor (who only cares about the financial return) and the environmentalist (who only cares about emissions reductions), because they suffer no disadvantage from ignoring the other variable.

However, it is still possible to determine a ranking using techniques derived from multi-objective optimization. Pareto ranking can be reasonably interpreted as representing cost-effectiveness, but caution is needed. If Pareto ranking is applied to the positive cost regime, where the standard metric $M_{s t d}$ is valid, the two are not always consistent. There is no problem where one measure dominates another in a Pareto sense: it is easy to show that this is precisely consistent with the standard 
metric. But as pointed out in Section 4, the same is not necessarily true for measures in two adjacent Pareto rankings. So the Pareto ranking is better seen as a reasonable approximation to the standard metric than a precise replacement.

More generally, the use of Pareto ranking means that all is not lost for the concept of cost-effectiveness under negative-cost conditions. But it is worth noting that a fine distinction is being made: no metric for cost-effectiveness can exist, but a ranking in order of cost-effectiveness is possible. A corollary is that both $c$ and $g$ need to be quoted to allow the ranking of measures to be properly assessed.

\subsection{Use of carbon price}

The analysis in Section 3 demonstrates that, for profit-making measures, no costeffectiveness metric is possible that satisfies the reasonable requirements 1 and 2 . But it is interesting to consider one metric, excluded because it fails to satisfy Requirement 2, because it raises some interesting points. The metric of interest is of the form

$$
M=f(c-P g)
$$

where $P$ is a constant. The reason for its exclusion is clear: $c$ and $g$ are not proportional, so rankings produced by it would be contestable in the same way as for equation (2). But there is a difference here, as can be seen by considering the constant. For the equation to be dimensionally homogeneous, $P$ must have the units $£ / t C$ or equivalent; that is, it is a carbon price. So this metric values emissions savings as cost savings and combines them with any return on investment. If the relationship between emissions savings and money embodied in the carbon price 
satisfied all parties, including the investors and environmentalists referred to earlier, then the failure of equation (3) to satisfy Requirement 2 would no longer be a problem. But such an eventuality seems remote, and for that reason the metric remains contestable and so fundamentally unsuitable. This is an important point, because such a metric would otherwise present a possible solution to the problem studied in the present paper.

The metric represented by equation (3) has other disadvantages. Most seriously, it requires an extra input - a carbon price must be specified before it can be deployed. Such external dependence would be unhelpful, especially if the carbon price were volatile or uncertain. In addition, the lack of salience of carbon price in everyday life means there is a risk that a metric based upon it might lack impact and credibility.

\subsection{Consequences for existing results}

One aspect of the analysis of the Toke and Taylor data in Section 5.1 that affects both the application of the standard metric and the results of the Pareto ranking is the appearance in many measures of the same value of $g$, namely $124.1 \mathrm{kgC} / \mathrm{MWh}$. It appears frequently because many of the measures are directed at reducing the consumption of electricity, of which this was the emissions factor at the time.

The relative ranking by the standard metric $M_{s t d}$ of a set of measures with the same emissions factor will be correct, precisely because the emissions factor is playing no role and only the cost of saving energy is being taken into account.

As shown in Section 5.1, the large number of measures directed at reducing electricity consumption in the study by Toke and Taylor (2007) means that only a small number of measures were incorrectly ranked out of the twelve originally 
considered. At the same time, the Pareto ranking works well for such a set because an unambiguous order can be determined: each Pareto front contains only one measure. As more measures with different emissions savings are introduced, the risk of incorrect rankings from the standard metric will increase but so too will the risk of a less discriminating Pareto ranking.

In the absence of detailed data, the impact on existing MACCs of the findings of the present work is unclear. But it seems likely that there will be ranking errors for measures with similar values of (negative) cost-effectiveness but different emissions savings.

\subsection{Future representations of cost-effectiveness}

The discussion above demonstrates that incorrect results can be obtained if measures that make a return on investment are ranked using the standard metric $M_{s t d}$. One possible solution would be to use Pareto ranking for all measures. This would have the advantage of simplicity due to a single assessment method, but there are significant disadvantages. Most importantly, as pointed out in Section 6.2, the Pareto ranking is not entirely consistent with the standard metric in the positive-cost regime, so such a change would be a retrograde step. In addition, Pareto ranking is less discriminating than the standard metric, with significant potential for equal rankings of measures as in Table 1. And finally, the division between negative- and positivecost measures would be invisible to Pareto ranking, allowing the possibility of positive-cost measures being ranked ahead of negative.

One option for ranking emissions reduction measures in the future would be to present the results in a standard MACC (as depicted in Fig. 1), by plotting the costeffectiveness value as before but shifting the horizontal position of the negative-cost 
blocks so that the Pareto ranking is shown. This would have several advantages, of which continuity is the main one. The standard MACC also has the great merit of presenting all the measures together in a single ranking. Another advantage is that the amount of money involved in the implementation of each measure would continue to be correctly represented by the areas of the blocks. Finally, the single MACC provides an effective vehicle for demonstrating overall effects such as changes in discount rates.

But there would be several disadvantages. A practical one is that equal Pareto ranking would not be visible in the diagram as it stands, and modifications would be needed, such as creating combined measures. This, however, would also be needed in other representations as will be shown later. Another disadvantage is potential confusion because measures are not ranked according to their cost-effectiveness. Most importantly, the present work has demonstrated that the value of $£ / t C$ determined for profit-making measures is not a cost-effectiveness, so its continued use in such a diagram would be misleading.

In view of these issues, there is a case for presenting the profit-making and lossmaking measures separately. The MACC could continue to be used for measures with positive costs, while a new presentation method would be needed for negative costs. 
Table 2. Pareto ranking of the negative-cost measures of Toke and Taylor (2007)

\begin{tabular}{|c|l|c|}
\hline \multirow{2}{*}{ Ranking } & \multicolumn{1}{|c|}{ Measure } & $\begin{array}{c}\text { Available reduction } \\
\text { (Mt C over 15 years) }\end{array}$ \\
\hline 1 & A. Domestic lighting & 4.64 \\
\hline 2 & B. Reduction of standby drain & 5.46 \\
\hline 3 & C. Savaplugs & 2.28 \\
\hline 4 & F. Compressed air leaks & 2.30 \\
\hline \multirow{2}{*}{$5=$} & D. No new electric heating & 9.26 \\
\cline { 2 - 3 } & H. Non-domestic lighting & 11.79 \\
\hline 7 & J. Motors & 23.83 \\
\hline 8 & K. Domestic insulation & 9.56 \\
\hline 9 & L. BEMS & 17.51 \\
\hline 10 & N. CHP & 19.52 \\
\hline
\end{tabular}

Since the Pareto ranking does not involve the calculation of a figure of merit, an appropriate ranking method would provide a list of the measures in ranking order along with the emissions reduction available.

The data for the negative-cost measures of Toke and Taylor (2007) have been presented in this form in Table 2. Note that the additional measures (with superscript "a") have been removed for consistency with the original data.

The data in Table 2 have been plotted as a stacked bar chart in Figure 6 to give a graphical representation of the Pareto ranking. It is arranged so that the ranking starts on the left, echoing the MACC. It would be possible to go further and form a hybrid diagram with such a bar chart for negative cost measures connected to a conventional MACC for positive cost. The advantages would be continuity and the ability to present a single ranking of all measures. But careful design would be needed to avoid both confusion and an inelegant appearance.

The main disadvantage of such separate charts is that in some cases it would take only a small change in the initial conditions (e.g. as part of a sensitivity analysis) to 
change the financial outcome of a measure from a negative to a positive NPV, thus requiring the use of the alternative ranking method and preventing meaningful comparison with the original value. There is no obvious solution to this problem other than to avoid such comparisons and perform separate sensitivity analyses for the two cases.

It would also be more difficult to demonstrate the impacts of certain wide scale changes, in costs for example, by shifting the whole MACC up or down. But the problems demonstrated with the existing method mean that there is no choice unless a better alternative ranking method is put forward.

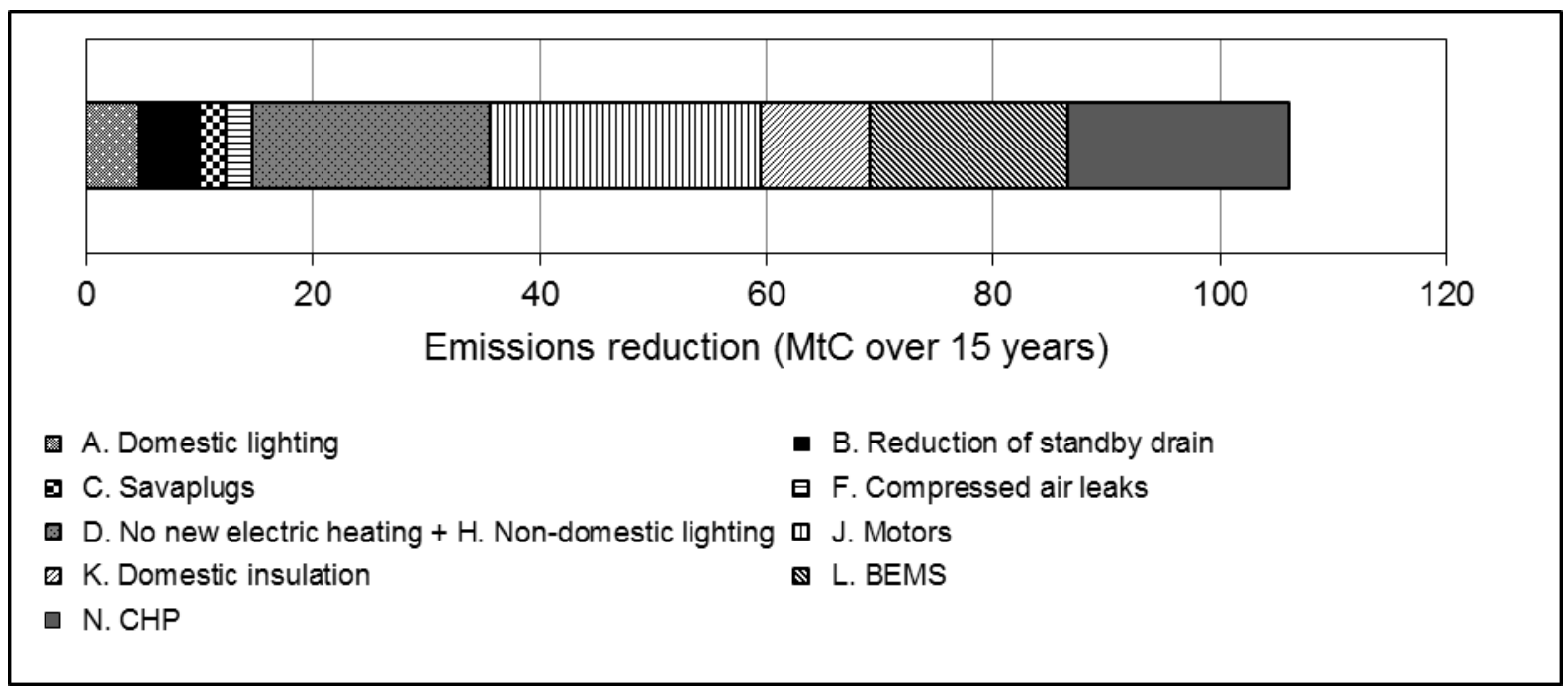

Figure 6. Graphical representation of the Pareto ranking of the data of Toke and Taylor

\subsection{Why was the issue not resolved earlier?}

Given the significant failings uncovered in the metric $M_{\text {std }}$ when applied to measures which make a profit, it seems surprising that the issue was not resolved earlier. The 
problem addressed in this paper was identified soon after the introduction of MACCs by Wallis (1992b), who pointed out that when the cost is negative, smaller emissions savings "appear preferred, being given higher ranking" (p. 1130). However, that comment formed part of a wider discussion (Wallis 1992a; Jackson 1992, 1993) and its importance appears not to have been recognized.

One possible clue as to why the issue did not resurface is the finding discussed above, that of all of the measures listed in the work by Toke and Taylor (2007), only a small number were incorrectly ranked by the standard metric $M_{\text {std }}$.

Two factors contributed to this outcome. First, as discussed in Section 6.4, the specific emissions saving is often the same for large groups of measures, in which case the metric depends only on the specific cost and so provides the correct ranking for that set. Second, even when the specific emissions saving does change between two measures, the perverse effect is often masked by a bigger change in the specific cost as described in Section 5.1.

\section{Conclusions}

An analysis of the conventional method used for ranking the economics of emissions reduction measures shows that it is inapplicable to the measures of greatest interest those that make a financial return.

A significant consequence is that it is not appropriate to use cost-effectiveness, measured in $£ / t C$ or equivalent, for ranking emissions reduction measures that make a profit. 
A mathematical analysis shows that if reasonable requirements are imposed, no function exists that can be used as a figure of merit for ranking profit-making emissions reduction measures.

A ranking method based on Pareto principles when applied to measures studied in earlier work leads to a fairly clear ranking order and identifies incorrectly ranked measures. Approximate methods applied to previously published MACCs also reveal incorrect rankings.

There are likely to be ranking errors as a result of the inapplicability of the standard metric in other published MACCs, and re-examination is recommended.

Conventional MACCs are not appropriate for ranking profit-making measures. Pareto ranking provides a possible alternative.

\section{Acknowledgments}

I would like to thank Professor Richard Green of Imperial College, University of London and colleagues at Loughborough University and the Institute of Energy and Sustainable Development, De Montfort University, for helpful comments. I also wish to thank Mr Taofeeq Ibn-Mohammed for his question about ranking methods for emissions reduction measures that prompted the present paper. Finally, thanks are due to the Confederation of British Industry (www.cbi.org.uk) for permission to reproduce Fig. 4 and to Pion Ltd., London (www.pion.co.uk), publishers of Environment and Planning B (www.envplan.com), for permission to reproduce Fig. 5. 


\section{References}

Allcott, H. and Mullainathan, S., 2010. Behavior and energy policy. Science 327, 1204-1205.

Beaumont, N.J, Tinch, R., 2004. Abatement cost curves: a viable management tool for enabling the achievement of win-win waste reduction strategies? Journal of Environmental Management 71, 207215.

Blok, K., Worrell, E., Cuelenaere, R., Turkenburg, W., 1993. The cost effectiveness of $\mathrm{CO}_{2}$ emission reduction achieved by energy conservation. Energy Policy 21, 656-667.

Bloomberg, 2010. Carbon markets - North America - Research Note, Bloomberg New Energy Finance, January 2010. Available at: https://www.bnef.com/WhitePapers/download/25 (Accessed 26 Jan 2012)

CBI, 2007. Climate change: everyone's business. CBI, London. Available at: http://www.cbi.org.uk/media/1058204/climatereport2007full.pdf (Accessed 26 Jan 2012)

CCC, 2008. Committee on Climate Change. Building a low-carbon economy - the UK's contribution to tackling climate change, The Stationery Office, December 2008. Available at: http://www.theccc.org.uk/pdf/TSO-ClimateChange.pdf (Accessed 26 Jan 2012)

Creyts, J., Derkach, A., Nyquist, S., Ostrowski, K., Stephenson, J., 2007. Reducing U.S. greenhouse gas emissions: How much at what cost?, McKinsey \& Company. Available at: http://www.mckinsey.com/Client_Service/Sustainability/Latest_thinking/ /media/McKinsey/dotcom/ client_service/Sustainability/PDFs/Reducing\%20US\%20Greenhouse\%20Gas\%20Emissions/US_ghg final_report.ashx (Accessed 26 Jan 2012)

Deb, K., 2001. Multi-objective Optimization Using Evolutionary Algorithms, Wiley, Chichester, UK. DECC, 2009. Carbon Valuation in UK Policy Appraisal: A Revised Approach, Department of Energy and Climate Change. Available at: http://www.decc.gov.uk/en/content/cms/what_we_do/lc_uk/valuation/valuation.aspx (Accessed 26 Jan 2012) 
DECC, 2010. Energy consumption in the United Kingdom, Department of Energy and Climate Change website. http://www.decc.gov.uk/en/content/cms/statistics/publications/ecuk/ecuk.aspx (Accessed 26 Jan 2012)

DECC, 2011. The Green Deal and Energy Company Obligation Impact Assessment, Department of Energy and Climate Change website.

http://www.decc.gov.uk/assets/decc/11/consultation/green-deal/3603-green-deal-eco-ia.pdf (Accessed 26 Jan 2012)

Defra, 2011. Department for Environment, Food and Rural Affairs. Guidelines to Defra / DECC's GHG Conversion Factors for Company Reporting. Available at: http://archive.defra.gov.uk/environment/business/reporting/pdf/110819-guidelines-ghg-conversionfactors.pdf (Accessed 26 Jan 2012)

DTI, 2007. Meeting the energy challenge - a white paper on energy. Department of Trade and Industry, London. Available at: http://www.berr.gov.uk/files/file39387.pdf (Accessed 26 Jan 2012)

Enviros Consulting Ltd, 2006. Review and development of carbon dioxide abatement curves for available technologies as part of the Energy Efficiency Innovation Review, Department for Environment, Food and Rural Affairs, January 2006. Available at: http://www.decc.gov.uk/assets/decc/what $\% 20 \mathrm{we} \% 20 \mathrm{do} /$ supporting\%20 consumers/saving_energy/ana lysis/enviros-report.pdf (Accessed 26 Jan 2012)

EPA, 2006. Global Mitigation of Non-CO2 Greenhouse Gases, United States Environmental Protection Agency. Available at: http://www.epa.gov/climatechange/economics/downloads/GlobalMitigationFullReport.pdf (Accessed 26 Jan 2012)

Fonseca, C.M., Fleming, P.J., 1995. An overview of evolutionary algorithms in multiobjective optimization, Evolutionary Computation 3, 1-16.

Goldberg, D.E., 1989. Generic algorithms in search, optimization and machine learning. AddisonWesley, Reading, MA. 
Hazeldine, T., Clark, W., Deller, L., Paschos, V., 2010. A Marginal Abatement Cost Curve for NHS England, AEA. Available at: http://www.sdu.nhs.uk/documents/MACC Final_SDU and_AEA.pdf (Accessed 26 Jan 2012)

Hogg, D., Baddeley, A., Ballinger, A., Elliott, T., 2008. Development of Marginal Abatement Cost Curves for the Waste Sector, Eunomia Research \& Consulting. Available at: http://www.theccc.org.uk/pdfs/Eunomia\%20Waste\%20MACCs\%20Report\%20Final.pdf (Accessed 26 Jan 2012)

Jackson, T., Roberts, S., 1989. Getting out of the Greenhouse-An Agenda for UK Action on Energy Policy. Friends of the Earth, London.

Jackson, T., 1991. Least-cost greenhouse planning supply curves for global warming abatement. Energy Policy 19, 35-46.

Jackson, T., 1992. People in greenhouses... Energy Policy 20, 190-191.

Jackson, T., 1993. Greenhouse conjectures - and refutations. Energy Policy 21, 722-725.

Kesicki, F., 2010. Marginal Abatement Cost Curves for Policy Making - Expert-Based vs. ModelDerived Curves. 33rd IAEE International Conference Rio de Janeiro. Available at: http://www.homepages.ucl.ac.uk/ ucft347/Kesicki_MACC.pdf (Accessed 26 Jan 2012)

Kesicki, F., Ekins, P., 2012. Marginal abatement cost curves: a call for caution. Climate Policy 12, 219-236.

Kesicki, F., Strachan, N., 2011. Marginal abatement cost (MAC) curves: confronting theory and practice. Environmental Science and Policy 14, 1195-1204.

McKinsey, 2012. Greenhouse gas abatement cost curves. McKinsey and company website. http://www.mckinsey.com/clientservice/sustainability/Costcurves.asp (Accessed 26 Jan 2012)

Kok, R., Annema, J.A., van Wee, B., 2011. Cost-effectiveness of greenhouse gas mitigation in transport: A review of methodological approaches and their impact. Energy Policy 39 (2011) 77767793. 
Meier, A.K., 1982. Supply curves of conserved energy, PhD thesis, Lawrence Berkeley Laboratory, University of California, Berkeley.

Mills, E., Wilson, D., Johansson, T.B, 1991. Getting started: no-regrets strategies for reducing greenhouse gas emissions. Energy Policy 19, 526-542.

Morthorst, P.E., 1994. Constructing $\mathrm{CO}_{2}$ reduction cost curves: The case of Denmark. Energy Policy 22 (1994) 964-970.

Pout, C.H., 2000. N-DEEM: the national nondomestic buildings energy and emissions model.

Environment and Planning B: Planning and Design 27, 721-732.

Sitnicki, S., Budzinski, K., Juda, J., Michna, J., Szpilewicz, A., 1991. Opportunities for carbon emissions control in Poland. Energy Policy 19, 995-1002.

Spencer, C., Pittini, M., 2008. Building a marginal abatement cost curve (MACC) for the UK transport sector, Committee on Climate Change, March 2008. Available at:

http://www.theccc.org.uk/other_docs/Tech\%20paper\%20supply\%20side\%20FINAL.pdf (Accessed 26 Jan 2012)

SQW Energy, 2009. Research into a carbon reduction target and strategy for Higher Education in England, SQW Consulting. Available at: http://www.hefce.ac.uk/pubs/hefce/2010/10_01/10_01a.pdf (Accessed 26 Jan 2012)

Toke, D., Taylor, S., 2007. Demand reduction in the UK — with a focus on the non-domestic sector. Energy Policy 35, 2131-2140.

Wallis, M.K., 1992a. Greenhouse ranking of gas-fuelling. Energy Policy 20, 174-176.

Wallis, M.K., 1992b. Ranking of greenhouse gas abatement measures. Energy Policy 20, 1130-1133. 
Appendix A: Proof that no figure of merit satisfying reasonable requirements is possible for negative-cost measures

Let the general figure of merit be defined by

$$
M=f(c, g)
$$

where $c$ and $g$ have the same meanings as earlier. Requirement 1 defined in Section 3 is that the figure of merit should decrease when $c$ gets smaller and $g$ gets bigger; that is,

$$
\begin{aligned}
& \frac{\partial M}{\partial c}>0 \\
& \frac{\partial M}{\partial g}<0
\end{aligned}
$$

for all $g>0$.

Requirement 2, also defined in Section 3, is that it must be impossible for a preference to be expressed between measures with the same value of $M$. A necessary (though possibly not sufficient) condition is that $c$ and $g$ be in a fixed ratio, thus preventing a preference based on variation in their relative values. This means the metric must have the form

$$
M=f(c / g)
$$


The proof is carried out by evaluating the partial derivatives of (A4) and comparing with (A2) and (A3). For simplicity, let

$$
s=c / g
$$

so that

$$
M=f(s) .
$$

Now calculate the derivatives of (A6) with respect to $c$ and $g$ :

$$
\begin{gathered}
\frac{\partial M}{\partial c}=\frac{d M}{d s} \frac{\partial s}{\partial c}=\frac{d M}{d s}\left(\frac{1}{g}\right), \\
\frac{\partial M}{\partial g}=\frac{d M}{d s} \frac{\partial s}{\partial g}=\frac{d M}{d s}\left(-\frac{c}{g^{2}}\right) .
\end{gathered}
$$

From (A7), $\partial M / \partial c$ has the same sign as $d M / d s$ in the domain $g>0$. So to agree with (A2) it must be the case that

$$
\frac{d M}{d s}>0
$$

Using (A9) in (A8) gives

$$
\frac{\partial M}{\partial g}<0 \text { if } c>0
$$




$$
\frac{\partial M}{\partial g}>0 \text { if } c<0
$$

Comparison of (A10) and (A11) with (A3) shows that Requirement 1 is satisfied for $c>0$ but not for $c<0$.

So any function of the form of equation (A4) fails to satisfy Requirement 1 when $c<$ 0. But equation (A4) is the most general form of a figure of merit satisfying Requirement 2.

It follows that no possible function can satisfy both requirements, and therefore no figure of merit exists that is suitable for ranking emissions reduction measures that make a profit. 